\title{
Retrospective analysis of risk factors for deep infection in lower limb Gustilo-Anderson type III fractures
}

Taku Ukai* (D, Kosuke Hamahashi, Yoshiyasu Uchiyama, Yuka Kobayashi and Masahiko Watanabe

\begin{abstract}
Background: Open fractures are among the most severe injuries observed in orthopedic patients. Treating open fractures is difficult because such patients with infections may require multiple operations and amputations. Furthermore, only a few studies have focused on antibiotic prophylaxis in open fractures and evaluated how to cover lost soft tissue to increase the success rate of reconstruction. We evaluated the risk factors for deep infection in lower limb Gustilo-Anderson (G-A) type III fractures.
\end{abstract}

Materials and methods: This retrospective study investigated patients who underwent surgical procedures for lower limb G-A type III fractures between January 2007 and January 2017 at our institution. We enrolled 110 patients with 114 lower limb G-A type III fractures (77 G-A type IIIA fractures and 37 G-A type IIIB fractures) who were followed up for at least 2 years. We compared patients presenting infections with those without infections by assessing the following factors: severe contamination, diabetes, smoking, Injury Severity Scale, segmental fracture, location of fracture, G-A classification, damage control surgery, methods of surgery, timing of fixation, combination of antibiotics used, duration of antibiotic prophylaxis, timing of wound closure, and soft-tissue reconstruction failure.

Results: Eighteen fractures presented deep infections. Compared with patients without infections, patients developing infections differed significantly in terms of severe contamination $(P<0.01), \mathrm{G}-\mathrm{A}$ classification $(P<0.01)$, duration of antibiotic prophylaxis $(P<0.01)$, timing of wound closure $(P<0.01)$, and incidence of soft-tissue reconstruction failure $(P<0.01)$. Skin grafting was associated with significantly higher failure rates than muscle and free flap reconstructions $(P=0.04)$. Treatment with antibiotics was significantly longer in patients with drug-resistant bacterial infections than in those without infections $(P<0.01)$.

Conclusion: Early flaps rather than skin grafting should be used to cover G-A type IIIB fractures, because skin grafting resulted in the highest failure rate among soft-tissue reconstructions in open fractures. Longer duration of antibiotic use had a significant impact not only on deep infection rates but also on the presence of drug-resistant bacteria. These findings suggest that prolonged use of antibiotics should be avoided in cases of open fractures.

Level of evidence: Level IV retrospective observational study.

Keywords: Open fracture, Infection, Soft-tissue reconstruction, Drug-resistant bacteria

${ }^{*}$ Correspondence: taku115@is.icc.u-tokai.ac.jp

Department of Orthopaedic Surgery, Surgical Science, Tokai University

School of Medicine, 143 Shimokasuya, Isehara, Kanagawa 259-1193, Japan

\section{Introduction}

G-A type III open fractures have a high infection rate [1-3]. Once deep infection or osteomyelitis has occurred, patients may require multiple operations and may develop significant dysfunction at the site of injury. Risk factors for deep infection include diabetes [4-8] and smoking $[9,10]$. Specifically, G-A type III open fractures 
are associated with severe soft-tissue injuries [1]. In cases where open fractures are complicated by massive loss of soft tissue, patients often require soft-tissue reconstruction. Although it is desirable to manage an open fracture by restoration with a soft-tissue cover as quickly as possible, this is often complicated by the requirement for cooperation with plastic surgeons. Therefore, it is essential to determine when to cover injuries that have lost soft tissue and to choose the optimal methodology. Lack et al. reported that the infection rate was significantly lower in patients in whom the wound was closed within 5 days of the injury [7]. Nevertheless, only a limited number of reports have evaluated how to cover lost soft tissue to increase the success rate of reconstruction $[11,12]$. While treatment with antibiotics is recommended $24-72 \mathrm{~h}$ after wound closure [13], only a few reports have focused on antibiotic prophylaxis in open fractures [8,13-15]. Open fractures may prolong the need for antibiotic therapy because they are frequently complicated by pneumonia, urinary tract infections, use of an artificial respirator, as well as several other conditions [16]. The purpose of this study is to identify factors that increase the risk of deep infection in lower limb G-A type III fractures.

\section{Materials and methods}

\section{Study design and population}

Patients with open fractures who underwent surgery from 2007 to 2017 were retrospectively investigated. The patient's mean age was 44.5 years (range 18-84 years). Overall, 81 patients (84 total fractures) were men, and 29 patients (30 total fractures) were women. Eighteen fractures $(15.8 \%)$ were diagnosed with a concomitant deep infection, and 3 resulted in amputation as a consequence of the infection. The mechanisms of injury were as follows: traffic accidents, 88 fractures; falls, 16 fractures; and other trauma, 10 fractures. The mean follow-up period was 37 months (range 24-119 months), and the mean Injury Severity Score (ISS) was 13.7 (range 9-34).

The inclusion criteria were patients with lower limb open fractures of G-A type III who were followed up for at least 2 years after the surgical procedure. Exclusion criteria included open fractures of the upper limb and foot, closed fractures, and open fractures of G-A types I, II, and IIIC (Fig. 1).

\section{Fractures}

Fractures were classified as follows: 77 G-A type IIIA fractures and 37 G-A type IIIB fractures. The location of the fractures was as follows: femur in 21 fractures, tibia in 54 fractures, and ankle in 39 fractures. We evaluated open fractures using the modified G-A classification. We evaluated the soft-tissue condition rather than the length of the wound. We defined fractures without

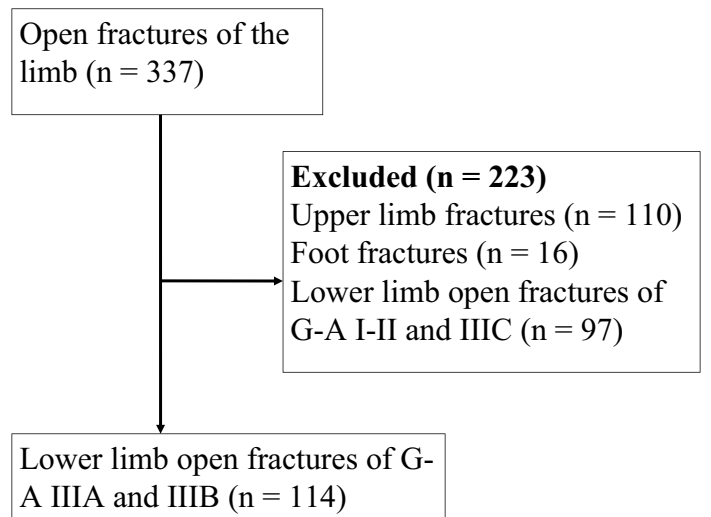

Fig. 1 Flow diagram showing patient identification and exclusion

severe contamination and extensive soft-tissue damage and those for which we could close the wounds as G-A type IIIA. Fractures with extensive soft-tissue damage and those for which we could not close the wounds were defined as G-A type IIIB (Table 1). We defined severe contamination as a wound that was contaminated with mud or sand.

\section{Criteria for deep infection}

Deep infection was diagnosed using recommendations from the Centers for Disease Control and Prevention [17]. In patients who were diagnosed with a deep infection, bacterial cultures were performed before debridement, during debridement, or both. Deep infection included a documented surgical site infection with bone involvement. The present study did not include patients with superficial infections. Pin tract infection not requiring surgery was also excluded.

\section{Treatment strategy}

The treatment strategy for open fractures adopted at the institution was as follows: open reduction and internal fixation (ORIF) was performed on the day of admission in patients with G-A type IIIA femur, tibia, and ankle fractures. Patients who had comminuted fractures, such as pilon and bicondylar tibial plateau fractures of G-A type IIIA, underwent external fixation as damage control surgery on the day of admission, and ORIF was performed after the soft-tissue swelling improved, as assessed by presence of skin wrinkling. Patients with open fractures of G-A type IIIB underwent external fixation as damage control surgery on the day of admission, and ORIF and soft-tissue reconstruction were performed after the soft-tissue swelling improved. Patients who presented with severe head, chest, or abdominal injury or had contraindications for surgery had their wounds 
Table 1 Modified Gustilo-Anderson classification

\begin{tabular}{|c|c|c|c|c|c|}
\hline Type & I & II & IIIA & IIIB & IIIC \\
\hline Wound size & $\leq 1 \mathrm{~cm}$ & $1-10 \mathrm{~cm}$ & About $5-10 \mathrm{~cm}$ & About $10 \mathrm{~cm}$ or longer & About $10 \mathrm{~cm}$ or longer \\
\hline Soft-tissue damage & Minimal & Minimal & Minimal or moderate & Severe & Severe \\
\hline Contamination & Clean & Clean or moderate & Clean or moderate & Moderate or severe & Moderate or severe \\
\hline Wound closure & Primary suture & Primary suture & Primary suture & Soft-tissue coverage & $\begin{array}{l}\text { Primary suture or soft- } \\
\text { tissue coverage }\end{array}$ \\
\hline Revascularization & Not needed & Not needed & Not needed & Not needed & Required \\
\hline
\end{tabular}

washed and their fractures fixed with splints in treatment rooms; they underwent ORIF or external fixation once their general condition improved.

\section{Soft-tissue reconstruction}

The mean time required for soft-tissue reconstruction was 13.7 days (range 0-31 days) for G-A type IIIB fractures. Prior to performing soft-tissue reconstruction, 20 fractures were treated with wet dressing and 17 patients were treated with negative-pressure wound therapy (NPWT). Twenty-six patients were treated with skin grafting only (femur: 2, tibia: 17, ankle: 7), 5 patients were treated with a muscle flap (tibia: 3 , ankle: 2 ), and 6 patients with a free flap (femur: 1 , tibia: 4 , ankle: 1 ). Skin grafting was performed as the only soft-tissue reconstruction method, and we attempted to completely cover the bones with soft tissue when performing skin grafting. During the skin grafting procedure, we meticulously checked whether the wounds were sufficiently covered with soft tissue and attempted to preserve the periosteum of the bone. Overall, 8 of 26 patients who received skin grafting underwent repeat operation. Of these eight patients, six were diagnosed with deep infections. Failure of soft-tissue reconstruction was considered in patients who underwent additional soft-tissue reconstruction after the first intervention.

\section{Antibiotics and culture}

A first-generation cephalosporin was administered for 96 fractures, while a combination of cephalosporin and aminoglycoside was administered for 18 fractures. For patients under $80 \mathrm{~kg}$, we used $1 \mathrm{~g}$ of a first-generation cephalosporin twice a day; for patients weighing over $80 \mathrm{~kg}$, we used $2 \mathrm{~g}$ of a first-generation cephalosporin twice a day. Aminoglycoside dosage was accompanied by therapeutic drug monitoring. In our institution, antibiotic withdrawal is decided by evaluating patients' fever, laboratory data (white blood cells and C-reactive protein), and wound discharge. The mean duration of antibiotic treatment was 11.9 days (range 2-34 days), and this period included only antibiotic prophylaxis. The antibiotic treatment duration was defined as the period of continuous use of antibiotics from the day of admission. This period did not include therapeutic antibiotic administration. Bacteria were identified in all patients diagnosed with a deep infection. The specific bacteria detected were as follows: methicillin-resistant Staphylococcus aureus (MRSA) in nine fractures, methicillinresistant staphylococci (MRS) in one fracture, and other bacteria in eight fractures (Table 2).

\section{Statistical analyses}

We evaluated age, body mass index, ISS, timing of fixation, duration of antibiotic prophylaxis, and timing of wound closure using the Mann-Whitney $U$ test. We used Fisher's exact test to evaluate severe contamination, diabetes, smoking status, segmental fracture, location of fracture, G-A classification, damage control surgery, methods of surgery, combinations of antibiotics, and soft-tissue reconstruction failure. We divided patients further into two groups: patients with resistant bacterial infections (10 fractures), and patients without resistant bacterial infections (8 fractures). The Mann-Whitney $U$ test was used to compare patients with and without antibiotic-resistant bacteria by evaluating the duration of antibiotic use and hospital stay. All tests were performed with a significance level of $P<0.05$.

\section{Results}

The comparison of fractures with and without deep infection revealed significant differences in terms of the presence of severe contamination (with infection $11.1 \%[2 / 18]$ versus without infection $4.2 \%$ [4/96], $P<0.01$ ), G-A fracture classification (infection rates:

\section{Table 2 Details of culture tests}

\begin{tabular}{ll}
\hline Isolated organism & Number \\
\hline Methicillin-resistant Staphylococcus aureus & 9 \\
Methicillin-resistant staphylococci & 1 \\
Other & 8 \\
\hline
\end{tabular}


IIIA $7.8 \%$ [6/77] versus IIIB 32.4\% [12/37], $P<0.01)$, duration of antibiotic prophylaxis (with infection: 14.1 days versus without infection: 11.2 days, $P<0.01$ ), timing of wound closure (with infection: 8.8 days versus without infection: 4 days, $P<0.01$ ), and soft-tissue reconstruction failure (failure rate: $21.6 \%, P<0.01$ ) (Table 3). Fisher's exact test also revealed that the occurrence of skin graft failure was significantly higher than that of muscle and free flap failure (failure rates: skin graft, $30.8 \%[8 / 26]$ versus muscle flap $0 \%[0 / 5]$, free flap $0 \%$ [0/6], $P=0.04$ ).

The Mann-Whitney $U$ test revealed that the duration of antibiotic prophylaxis was significantly longer in patients who had drug-resistant bacterial infections than in those who did not (19.8 versus 11.2 days, $P<0.01)$. Hospital stay duration was not significantly different $(P=0.73)$ between patients who had resistant bacteria (45.2 days) and those who did not (35.4 days).

\section{Discussion}

The findings of the comparison between the infection and noninfection groups revealed that severe contamination, G-A classification, duration of antibiotic prophylaxis, the timing of wound closure, and soft-tissue reconstruction failure had a substantial impact on deep infections. Skin grafts alone had significantly higher failure rates than muscle and free flap reconstructions. The antibiotic prophylaxis duration was significantly longer in patients who had drug-resistant bacterial infections than in those who did not.

Various factors related to the rates of deep infection in open fractures have been reported previously [4-10], including associated soft-tissue injury, fracture type, and treatment strategy. In particular, some types of open fracture such as gunshot and farmyard injuries and segmental fractures may influence deep infections. In this study, although there were no cases of gunshot or farmyard injuries, six patients had segmental fractures. Unlike other fractures, segmental fractures frequently entail severe soft-tissue injuries that induce deep infection.

Table 3 Comparison of fractures with and without infection

\begin{tabular}{|c|c|c|c|}
\hline Valuable & With infection & Without infection & $P$ value \\
\hline Age (years) & $47.8( \pm 19.4)$ & $43.1( \pm 19.7)$ & 0.41 \\
\hline Body mass index $\left(\mathrm{kg} / \mathrm{m}^{2}\right)$ & $24.4( \pm 4.8)$ & $23( \pm 4.9)$ & 0.18 \\
\hline Severe contamination & 5 & 5 & $<0.01$ \\
\hline Diabetes & 2 & 3 & 0.13 \\
\hline Smoking & 10 & 46 & 0.61 \\
\hline Injury Severity Scale & $16.8( \pm 7.0)$ & $13.2( \pm 7.2)$ & 0.06 \\
\hline Segmental fracture & 2 & 4 & 0.2 \\
\hline Location of fracture & & & 0.5 \\
\hline Femur & 4 & 17 & \\
\hline Tibia & 10 & 44 & \\
\hline Ankle & 4 & 35 & \\
\hline G-A classification & & & $<0.01$ \\
\hline$\| \mathrm{A}$ & 6 & 71 & \\
\hline$\| \mathrm{B}$ & 12 & 25 & \\
\hline Damage control surgery & $13 / 18$ & $58 / 96$ & 0.44 \\
\hline Methods of surgery & & & 0.11 \\
\hline Nail & 6 & 35 & \\
\hline Plate & 7 & 42 & \\
\hline External fixation & 3 & 4 & \\
\hline Pinning & 2 & 15 & \\
\hline Timing of fixation (days) & $9.1( \pm 6.6)$ & $8.5( \pm 7.3)$ & 0.3 \\
\hline Combination of antibiotics & & & 0.3 \\
\hline First-generation cephalosporin & 16 & 80 & \\
\hline First-generation cephalosporin + aminoglycoside & 3 & 15 & \\
\hline Antibiotic duration (days) & $14.1( \pm 7.7)$ & $11.2( \pm 5.9)$ & $<0.01$ \\
\hline Timing of wound closure (days) & $8.8( \pm 9.4)$ & $4( \pm 8.0)$ & $<0.01$ \\
\hline Soft-tissue reconstruction failure & 6 & 2 & $<0.01$ \\
\hline
\end{tabular}


However, there were no significant differences between patients with and without infection (Table 3). Regarding G-A classification, G-A type III fractures are reported to have a higher infection rate than G-A types I and II [18], with G-A type IIIB specifically having the highest rate of infection [17-19]. This suggests that G-A type III fractures frequently complicate severe soft-tissue injury, and as such, it is important to cover the skin injury as soon as possible. We found significant differences in the timing of primary wound closure and soft-tissue reconstruction failure, defined as multiple soft-tissue reconstruction surgeries and the resultant deep infection rate after open fracture. The timing of wound closure was longer for G-A type IIIB fractures than for G-A type IIIA fractures because G-A type IIIB fractures require meticulous softtissue reconstruction. Open fractures should be closed as soon as possible. Gopal et al. [12] stated that G-A type IIIB fractures should be covered in less than $72 \mathrm{~h}$. Most G-A type IIIB fractures require flaps; however, such surgery is long and entails the risk of flap necrosis. Thus, we performed skin grafting when bones were covered with muscle and soft tissue. The G-A classification is used for evaluating open fractures worldwide, and we classified open fractures according to this classification in this study. However, the G-A classification cannot evaluate muscle and soft-tissue damage. These factors may have affected infection, and our grouping of open fractures may have been inappropriate. Thus, we are evaluating open fractures prospectively by evaluating these factors.

According to British Association of Plastic, Reconstructive and Aesthetic Surgeons guidelines [20], open fractures should be covered within 5-7 days after injury. In this study, the timing of wound closure of the infection group was longer than that recommended, as some patients required NPWT to improve their wound conditions. Previous metaanalyses have concluded that NPWT not only reduces the infection rate but also reduces flap necrosis and flap revision rates [21, 22]. By contrast, the WOLLF trial demonstrated that there was no improvement in wounds with NPWT and that delays in covering the fracture should be avoided [23]. In this study, we valued the soft-tissue condition more than the interval between soft-tissue reconstruction and injury so as to improve the soft-tissue condition. However, delayed skin closure may affect infection rates. The results suggest that it is more important to cover a wound within 7 days than to use NPWT for over 7 days, so as to reduce infection.

Mathews et al. [24] reported that patients who underwent multiple reconstructive surgeries had higher infectious complication rates than those who underwent single-stage orthoplastic fixation and coverage (34.6\% versus $4.2 \%)$. Soft-tissue reconstruction failure is almost synonymous with the development of deep infection. We believe that soft-tissue reconstruction failure leads to the development of infection. As the skin prevents bacteria on our bodies from infiltrating the wound and soft-tissue reconstruction failure prolongs the period during which the skin barrier is missing, bacteria may easily infiltrate wounds. In this study, we meticulously checked wounds and attempted to preserve the bone periosteum. We covered bone with soft tissues sufficiently before performing skin grafting. However, skin grafting had the highest failure rate $(30.8 \%)$ among the methods of soft-tissue reconstruction. This suggests that skin grafting is insufficient for treating G-A type IIIB fractures. Although skin grafting is a less complex procedure to perform than a muscle flap or free flap intervention, it frequently fails at locations with thin soft tissue; For example, it has been reported that a fracture of the tibia has a $244 \%$ increased risk of a deep infection compared with a nontibial fracture [25]. In our study, 8 of 26 patients who underwent skin grafting required reoperation, and 6 of 8 patients were diagnosed with a deep infection. Typically, at our hospital, surgeons choose a skin graft when bones and tendons are sufficiently covered by muscle but choose muscle and free flap procedures when bones and tendons are not sufficiently covered by muscle tissue, in accordance with established institutional criteria. However, we may unintentionally choose soft-tissue reconstruction inappropriately instead of a muscle flap or free flap, because these procedures require longer operation times and are more difficult procedures than skin grafting.

There are a limited number of reports that provide sufficient evidence regarding the combination of antibiotics to be used or their route and duration of administration in open fractures. Redfern et al. compared a combination of cefazolin and gentamicin with piperacillin/tazobactam in G-A type III fractures [26]. They reported that there was no significant difference between treatments and the rate of infection. Lloyd et al. reported that the infection rate was slightly decreased when antibiotics specifically targeting Gram-negative bacteria were selected; however, there were no significant differences in the rate of osteomyelitis [27]. Based on these results, the authors recommended cefazolin or clindamycin in open fractures. However, other authors have recommended cefazolin plus aminoglycoside or ampicillin plus sulbactam [28-30]. In the present study, we used cefazolin plus aminoglycoside to cover Gram-negative bacteria when the wound was particularly contaminated. Interestingly, there was no significant difference between the cephalosporin group and the cephalosporin plus aminoglycoside group. This may be due to the retrospective nature of our study and that the combination of antibiotics used was based on the individual clinician's discretion. Therefore, 
an extensive investigation to determine the most efficacious combination of antibiotics is needed in future studies.

Regarding the duration of antibiotic use, Hoff et al. recommended 72-h administration of antibiotics within $24 \mathrm{~h}$ of wound closure in G-A type III [13]. Long-term antibiotic use can lead to drug-resistant bacteria [14, 31, 32]; in our case, drug-resistant bacteria were detected in over half of the patients with infections (Table 2). The occurrence of drug-resistant bacteria was also significantly higher with prolonged antibiotic administration (19.8 versus 11.6 days). This finding suggests that the duration of antibiotic therapy had a significant impact on deep infection. Our findings also suggest that long-term antibiotic administration should be avoided to decrease the rate of drug-resistant bacterial infections.

This study has several limitations. First, multiple factors affect the occurrence of deep infection after open fractures, such as degree of soft-tissue injury and timing of antibiotic therapy. However, given the retrospective nature of this study, we could not evaluate these factors. Second, we could not evaluate potential risk factors associated with open fractures using logistic regression analysis due to the lack of an adequate sample size. Thus, we are currently gathering data related to open fractures to increase the sample size for a future analysis. Third, we could not control the combination of antibiotics used or their duration of administration. Fourth, we could not evaluate contamination of the wound quantitatively. Previous studies have reported that a contaminated wound increases the deep infection rate [25, 26, 33]. Nevertheless, there are no standard criteria for determining whether a wound is severely contaminated with mud, sand, or seawater; therefore, additional experimentation is needed in the future to evaluate these criteria. Fifth, we used the G-A classification to evaluate open fractures, which is widely accepted by many researchers. Nevertheless, it has been pointed out that, despite the fact that G-A type IIIB includes a wide range of open injuries, there are only two categories of severe fractures (G-A types IIIB and IIIC), and the reliability and reproducibility of this classification have been demonstrated to be suboptimal. The Classification and Outcomes Committee of the Orthopaedic Trauma Association (OTA) was created to overcome the problems with the G-A classification. Agel et al. [34] reported that the OTA classification demonstrated moderate to excellent interobserver reliability. This classification consists of five factors: skin injury, muscle injury, arterial injury, contamination, and bone loss. Nevertheless, we could not evaluate muscle and contamination efficiently because of the retrospective nature of this study. Sixth, antibiotic withdrawal was at the discretion of each orthopedic surgeon, and this may have affected the results.

In conclusion, we believe that skin grafting should not be applied to G-A type IIIB fractures while early flap coverage should be the preferred option. Antibiotic treatment duration had a significant impact not only on deep infection rates but also on the presence of drug-resistant bacteria. These data suggest that prolonged antibiotic use should be avoided in cases of open fractures.

\section{Abbreviations \\ G-A: Gustilo-Anderson; ORIF: Open reduction and internal fixation; MRSA: Methicillin-resistant Staphylococcus aureus; MRS: Methicillin-resistant staphylococci.}

\section{Acknowledgements}

None.

\section{Authors' contributions}

T.U. designed the study; K.H., Y.U., Y.K., and M.W. collected data; T.U. and K.H. made the major contributors to writing the manuscript. All authors read and approved the final manuscript.

\section{Funding}

None.

Availability of data and materials

Not applicable.

\section{Ethics approval and consent to participate}

This study was approved by the Ethics Committee of Tokai University. The authors state that this study conforms to the ethical standards laid down in the most recent version of the Declaration of Helsinki. All patients were informed about the study and consented to participate.

\section{Consent for publication}

All patients gave consent for publication.

\section{Competing interests}

The authors declare that they have no competing interests.

Received: 11 January 2020 Accepted: 7 May 2020

Published online: 18 July 2020

References

1. Westgeest J, Weber D, Dulai SK, Bergman JW, Buckley R, Beaupre LA (2015) Factors associated with development of nonunion or delayed healing after an open long bone fracture: a prospective cohort study of 736 subjects. J Orthop Trauma 30:149-155

2. Harley BJ, Beaupre LA, Jones CA, Dulai SK, Weber DW (2002) The effect of time to definitive treatment on the rate of nonunion and infection in open fractures. J Orthop Trauma 16:484-490

3. Papakostidis C, Kanakaris NK, Pretel J, Faour O, Morell DJ, Giannoudis PV (2011) Prevalence of complications of open tibial shaft fractures stratified as per the Gustilo-Anderson classification. Injury 42:1408-1415

4. Molina CS, Stinner DJ, Fras AR, Evans JM (2015) Risk factors of deep infection in operatively treated pilon fractures (AO/OTA:43). J Orthop 12:S7-13

5. Ovaska MT, Madanat R, Mäkinen TJ (2016) Predictors of postoperative wound necrosis following primary wound closure of open ankle fractures. Foot Ankle Int 37:401-406

6. Lawing CR, Li FC, Dahners LE (2015) Local injection of aminoglycosides for prophylaxis against infection in open fractures. J Bone Jt Surg Am 97:1844-1851 
7. Lack WD, Karunakar MA, Angerame MR, Seymour RB, Sims S, Kellam JF, Bosse MJ (2015) Type III open tibia fractures: immediate antibiotic prophylaxis minimizes infection. J Orthop Trauma 29:1-6

8. Zumsteg JW, Molina CS, Lee DH, Pappas ND (2014) Factors influencing infection rates after open fractures of the radius and/or ulna. J Hand Surg Am 39:956-961

9. Donigan JA, Fredericks DC, Nepola JV, Smucker JD (2012) The effect of transdermal nicotine on fracture healing in a rabbit model. J Orthop Trauma 26:724-727

10. Castillo RC, Bosse MJ, MacKenzie EJ, Patterson BM (2005) Impact of smoking on fracture healing and risk of complications in limb-threatening open tibia fractures. J Orthop Trauma 19:151-157

11. Fowler T, Whitehouse M, Riddick A, Umraz Khan, Kelly M (2019) A retrospective comparative cohort study comparing temporary internal fixation to external fixation at the first stage debridement in the treatment of type IIIB open diaphyseal tibial fractures. J Orthop Trauma 33:125-130

12. Gopal S, Majumder S, Batcherlor AGB, Knight SL, Smith RM (2000) Fix and flap: the radical orthopaedic and plastic treatment of severe open fractures of the tibia. Bone Joint J 82:959-966

13. Hoff WS, Bonadies JA, Cachecho R, Dorlac WC (2011) East Practice Management Guidelines Work Group: update to practice management guidelines for prophylactic antibiotic use in open fractures. J Trauma 70:751-754

14. Al-Arabi YB, Nader N, Hamidian-Jahromi AR, Woods DA (2007) The effect of the timing of antibiotics and surgical treatment on infection rates in open long-bone fractures: a 9-year prospective study from a distinct general hospital. Injury 38:900-905

15. Leonidou A, Kiraly Z, Gality H, Apperley S, Vanstone S (2014) The effect of the timing of antibiotics and surgical treatment on infection rates in open long-bone fractures: a 6-year prospective study after a change in policy. Strategies Trauma Limb Reconstr 9:167-171

16. Moloney GB, Pan T, Van Eck CF, Patel D, Tarkin I (2016) Geriatric distal femur fracture: are we underestimating the rate of local and systemic complications? Injury 47:1732-1736

17. Centers for Disease Control and Prevention. CDC/NHSN Surveillance Definitions for Specific Types of Infections. http://www.cdc.gov/nhsn/ pdfs/pscmanual/17pscnosinfdef_current.pdf. Accessed 24 Feb 2019

18. Gustilo R, Mendonza R, Williams D (1984) Problems in the management of type III (severe) open fractures: a new classification of type III open fractures. J Trauma 24:742-746

19. Matos MA, Lima LG, Oliveira LAA (2015) Predisposing factors for early infection in patients with open fractures and proposal for a risk score. J Orthop Traumatol 16:195-201

20. Trickett RW, Rahman S, Page P, Pallister I (2015) From guidelines to standards of care for open tibial fractures. R Coll Surg Eng 97:469-475

21. Liu X, Zhang H, Cen S, Huang F (2018) Negative pressure wound therapy versus conventional wound dressing in treatment of open fractures: a systematic review and meta-analysis. Int J Surg 53:72-79

22. Arti H, Khorami M, Ebrahimi-Nejad V (2016) Comparison of negative pressure wound therapy (NPWT) and conventional wound dressing in open fracture wounds. Pak J Med Sci 32:65-69
23. Costa ML, Achten J, Bruce J, Tutton E, Petrou S, Lamb SE, Parsons NR (2018) Effect of negative pressure wound therapy vs standard wound management on 12-month disability among adults with severe open fracture of the lower limb: the WOLLF randomized clinical trial. JAMA 319:2280-2288

24. Mathews JA, Ward J, Champman TW, Khan UM, Kelly MB (2015) Singlestage orthoplastic reconstruction of Gustilo-Anderson grade III open tibial fractures greatly reduces infection rates. Injury 46:2263-2266

25. Hull PD, Johnson SC, Stephen DJG, Kreder HJ, Jenkinson RJ (2014) Delayed debridement of severe open fractures is associated with a higher rate of deep infection. Bone Joint J 96B:379-384

26. Redfern JP, Wasilko SM, Groth ME, McMillian WD, Bartlett CS (2016) Surgical site infections in patients with type 3 open fractures: comparing antibiotic prophylaxis with cefazolin plus gentamicin versus piperacillin/ tazobactam. J Orthop Trauma 30:415-419

27. Lloyd BA, Murray CK, Shaikh F, Carson ML, Blyth DM, Schnaubelt ER, Whitman TJ, Tribble DR (2017) Early infectious outcomes after addition of fluoroquinolone or aminoglycoside to posttrauma antibiotic prophylaxis in combat-related open fracture injuries. J Trauma Acute Surg 83:854-861

28. Patzakis MJ, Harvey JP Jr, Ivler D (1974) The role of antibiotics in the management of open fractures. J Bone Joint Surg Am 56:532-541

29. Braun R, Enzler MA, Rittmann WW (1987) A double-blind clinical trial of prophylactic cloxacillin in open fractures. J Orthop Trauma 1:12-17

30. Hanretty AM, Gallagher JC (2018) Shortened courses of antibiotics for bacterial infections: a systematic review of randomized controlled trials. Pharmacotherapy 38:674-687

31. Bryson DJ, Morris DLJ, Shivji FS, Rollins KR, Snape S (2016) Antibiotic prophylaxis in orthopaedic surgery: difficult decisions in an era of evolving antibiotic resistance. Bone Joint J 98B:1014-1019

32. Baur D, Gladstone BP, Burkert F, Carrara E, Foschi F, Döbele S, Tacconelli PE (2017) Effect of antibiotics stewardship on the incidence of infection and colonisation with antibiotic-resistant bacteria and Clostridium difficile infection: a systematic review and meta-analysis. Lancet Infect Dis 17:990-1001

33. Yokoyama K, Itoman M, Nakamura K, Uchino M, Nitta H (2009) New scoring system predicting the occurrence of deep infection in open upper and lower extremity fractures: efficacy in retrospective re-scoring. Arch Orthop Trauma Surg 129:469-474

34. Agel J, Evans AR, Marsh JL, Decoster TA, Lundy DW, Kellam JF, Jones CB, DeSilva GL (2013) The OTA open fracture classification: a study of reliability and agreement. J Orthop Trauma 27:379-384

\section{Publisher's Note}

Springer Nature remains neutral with regard to jurisdictional claims in published maps and institutional affiliations.

\section{Submit your manuscript to a SpringerOpen ${ }^{\circ}$ journal and benefit from:}

- Convenient online submission

- Rigorous peer review

- Open access: articles freely available online

- High visibility within the field

Retaining the copyright to your article

Submit your next manuscript at springeropen.com 